\title{
Percepção da Equipe de Enfermagem do Hospital Universitário de São Luís do Maranhão Frente ao Ambiente e Segurança no Trabalho
}

\section{Perception of the University Hospital Nursing staff of São Luís of Maranhão Regarding the Environment and Safety at Work}

\author{
Helone Eloísa Frazão Guimarães Faray*a; Rosemary Matias`; Everton Ferreira Lemos ${ }^{\mathrm{b}}$; Arlindo Faray Vieirac; \\ Eduardo de Castro Ferreira ${ }^{a}$ \\ a'Universidade Anhanguera-Uniderp, Programa de Pós-Graduação Stricto Sensu em Meio Ambiente e Desenvolvimento Regional. MS, Brasil. \\ bUniversidade Federal do Mato Grosso do Sul. MS, Brasil. \\ 'Faculdade Fucap Univinte, Programa de Pós-Graduação Stricto Sensu em Ciências Contábeis. SC, Brasil. \\ *E-mail: helonefaray@hotmail.com
}

\begin{abstract}
Resumo
Os profissionais da equipe de enfermagem realizam, em seu cotidiano de trabalho, muitas atividades laborais que conduzem a riscos ocupacionais relacionados aos resíduos biológicos, entre outros, e que precisam ser levados em consideração para que o ambiente de trabalho seja vivenciado com segurança. Destarte, objetivou-se demonstrar a percepção da equipe de enfermagem frente ao ambiente e segurança no trabalho. Tratase de uma pesquisa de natureza aplicada com abordagem quantitativa e qualitativa de caráter descritivo exploratório, transversal, realizada no Hospital Universitário em São Luís do Maranhão, em que a equipe de enfermagem é composta por enfermeiros, técnicos e auxiliares de enfermagem. Este estudo foi realizado por meio da aplicação de questionários a 98 profissionais de enfermagem, que compõem a unidade de saúde da criança do Hospital Universitário em São Luís do Maranhão, de acordo com o critério de inclusão adotado. Sobre as atividades que as equipes de enfermagem consideram mais perigosas para a sua saúde e para o ambiente hospitalar, o maior percentual está para o descarte de resíduo hospitalar (33,7\%), seguido da manipulação dos resíduos dos pacientes $(18,4 \%)$ e aspiração traqueal (18,4\%). A prevenção e habilidades em educação e saúde devem ser assimiladas entre profissionais de enfermagem, sobre o manejo adequado de resíduos e incentivar a implementação de precauções para minimizar a ocorrência de agravos, que comprometem a saúde e atividades laborais desenvolvidas no seu ambiente, bem como orientações sobre as consequências dos acidentes de trabalho.
\end{abstract}

Palavras-chave: Enfermagem. Resíduos Biológicos. Ambiente Hospitalar.

\begin{abstract}
The nursing team professionals carry out many work activities in their daily work that lead to occupational risks related to biological waste, among others, and that need to be taken into account so that the work environment is experienced safely. Thus, the objective was to demonstrate the nursing team perception regarding the environment and safety at work. It is an applied nature research with a quantitative and qualitative approach of an exploratory, transversal descriptive character, carried out at University Hospital in São Luís do Maranhão, where the nursing team is composed of nurses, technicians and nursing assistants This study was carried out by applying questionnaires to 98 nursing professionals who make up the child health unit at university hospital in São Luís do Maranhão, according to the inclusion criterion adopted. Regarding the activities that the nursing teams consider as the most dangerous for their health and for the hospital environment, the highest percentage is for the disposal of hospital waste (33.7\%), followed by the handling of patient waste (18.4\%) and tracheal aspiration $(18.4 \%)$. Prevention and skills in education and health must be assimilated among nursing professionals, on the proper waste management and encourage the implementation of precautions to minimize the injuries occurrence that affect the health and work activities carried out in their environment, as well as guidance on the consequences of accidents at work.
\end{abstract}

Keywords: Nursing. Biological Waste. Hospital Environment.

\section{Introdução}

Para que o processo de trabalho possa acontecer, de forma segura, são necessárias condições adequadas relacionadas ao ambiente, à equipe, bem como ao material relacionado ao desempenho das atividades. O processo de trabalho de enfermagem segue a divisão do trabalho baseada no modo operatório, em que o enfermeiro utiliza seu saber como instrumental ideológico de poder. Essa divisão técnica caminha no mesmo sentido da divisão social do trabalho e é, portanto, um trabalho fracionado e fragmentado (FURTADO; ARAÚJO JÚNIOR, 2010).

$\mathrm{Na}$ prática assistencial, a cultura de segurança resulta de um complexo sistema que necessita de políticas, de programas e de apoio dos gestores aos profissionais que estão diretamente envolvidos com os pacientes, para que tenham condições de desenvolver uma assistência segura em todas as etapas do processo (GASPARINO et al., 2017) e, o primeiro passo para elaborar medidas que venham a prevenir, amenizar e se possível sanar os riscos, é conhecê-los (MARTINS et al., 2020).

A atividade profissional, no setor hospitalar, é considerada uma das mais perigosas à saúde do trabalhador, uma vez que está relacionada a um grande número de doenças ocupacionais e acidentes de trabalho (ROCHA et al., 2004) e, os profissionais da enfermagem são a classe mais afetada, tornando-os mais propensos a doenças específicas, causadas por fatores físicos, químicos, biológicos, mecânicos e 
psicossociais (PUCCI et al., 2017; MARTINS et al., 2020). Nesse sentido, é muito importante que a cultura de segurança, na qual todos os trabalhadores, incluindo profissionais envolvidos no cuidado e gestores, assumam responsabilidade pela sua própria segurança, pela segurança de seus colegas, pacientes e familiares, seja adotada e estimulada (SILVABATALHA; MELLEIRO, 2015; BRASIL, 2017; SOARES et al., 2019).

$\mathrm{O}$ uso de EPIs aliado às medidas de proteção coletivas são essenciais na prevenção de acidentes e patologias na equipe de enfermagem, uma vez que se enquadra no grupo de maior exposição aos riscos ocupacionais em função do contato direto com o paciente, com agulhas e diferentes tipos de perfuro cortantes, equipamentos, soluções e situações, que implicam na grande possibilidade de contato com sangue e outros fluidos orgânicos contaminados por uma variedade de patógenos desencadeadores de doenças (NISHIDE et al., 2004). A utilização de ferramentas, como: luvas de látex, touca e máscara, é importante e imprescindível para proteção do trabalhador e dos produtos manipulados (SOARES et al., 2019).

No entanto, os EPIs não descartam a possibilidade de ocorrerem acidentes, pois os agentes químicos, físicos, biológicos ainda permanecem no ambiente de trabalho. A utilização dos equipamentos de proteção individual tende a reduzir a probabilidade de dano e minimizam as consequências (TALHAFERRO et al., 2008). Informar e educar as equipes para que sigam as medidas admissíveis para manter a segurança nas organizações, além de oferecer condições que minimizem os riscos cabíveis de prevenção, que possam oferecer danos à saúde de seus colaboradores é responsabilidade das instituições (SOARES et al., 2019). Porém, o descumprimento das recomendações para utilização de barreiras de proteção pela equipe de enfermagem é uma realidade (SARQUIS; FELLI, 2009).

De acordo com Bakke e Araújo (2010), o trabalhador, quando orientado, pode evitar as situações de risco e a administração pode direcionar e adaptar medidas mitigatórias de risco à realidade desses profissionais. A reciclagem de hábitos profissionais consolidados, pela prática diária, incluindo o treinamento sobre o uso correto de equipamentos de proteção individual e adoção de precauções, em todas as etapas da assistência, é uma necessidade presente (SOARES et al., 2019).

Por fazer parte dessa realidade e diante da necessidade da presença constante do enfermeiro junto à clientela dentro dos serviços de saúde, e da indiscutível essencialidade do seu trabalho, se faz necessário, na medida do possível, que o ambiente de trabalho seja seguro. Este estudo objetivou demonstrar a percepção da equipe de enfermagem do Hospital Universitário de São Luís do Maranhão frente ao ambiente e segurança no trabalho.

\section{Material e Métodos}

\subsection{Local e tipo de estudo}

Trata-se de uma pesquisa de natureza aplicada com abordagem quantitativa e qualitativa de caráter descritivo exploratório, transversal, realizada no Hospital Universitário em São Luís do Maranhão. A equipe de enfermagem é composta por enfermeiros, técnicos e auxiliares de enfermagem.

A escolha dessa população atende aos objetivos da pesquisa, uma vez que está diretamente envolvida com o manejo dos resíduos sólidos dos serviços de saúde (RSSS) e por ser a categoria mais numerosa no conjunto dos trabalhadores da saúde. Consequentemente, aquela que mais executa procedimentos hospitalares e tende a produzir maior quantidade de resíduos. Foi escolhida toda a equipe de enfermagem da Unidade de Atenção à Criança, que é composta pelo setor da internação pediátrica e UTI pediátrica (UTI PED).

Esta pesquisa foi realizada no Hospital Universitário, nas dependências da Unidade de Atenção à Criança (internação pediátrica) composta por três alas: Ala A, na qual se encontram internados e os pacientes cirúrgicos, Ala $\mathrm{D}$, em que ficam os pacientes com doenças infectocontagiosas, e Ala E, na qual estão os pacientes com patologias diversas, bem como na UTI PED. Quanto à equipe de enfermagem, um total de 23 (23,5\%) são enfermeiros, 63 (64,3\%) técnicos em enfermagem e $12(12,2 \%)$ auxiliares de enfermagem. Totalizando uma população de 131 profissionais de enfermagem, destes, 98 funcionários participaram da pesquisa de acordo com os critérios de inclusão adotados.

\subsection{Critérios de inclusão}

Os critérios de inclusão da equipe de enfermagem foram: atuar na Unidade de Atenção à Criança (ALA A, ALA D, ALA E e UTI PED), estar presente no setor no dia da entrega do instrumento de coleta de dados, participar voluntariamente da pesquisa, após lerem e assinar o Termo de Consentimento Livre e Esclarecido (Apêndice B). Cabe ressaltar que embora a participação na pesquisa envolvesse riscos mínimos, como o de constrangimento ou de exposição frente à possibilidade de vazamento dos dados, a coleta de dados, bem como o arquivamento dos mesmos foi realizado de modo a atender aos preceitos e recomendações da Resolução n ${ }^{\circ}$ 466/2012 (BRASIL,2012), que determina as diretrizes e normas regulamentadoras de pesquisas envolvendo seres humanos, garantindo o sigilo, o anonimato e a confidencialidade dos participantes. Entre os benefícios estão a reflexão e a orientação dos trabalhadores às situações de risco, de modo a minimizar os riscos a que os profissionais de enfermagem estão expostos no ambiente de trabalho.

\subsection{Coleta de dados}

Os dados foram coletados juntos aos profissionais de enfermagem, por meio de questionário composto por dez 
questões (Apêndice A), sendo constituído de perguntas abertas e fechadas. A coleta de dados foi realizada nos meses de julho a setembro do ano de 2018 .

\subsection{Tratamento dos dados e análise estatística}

Os dados foram tabulados no Microsoft Office Excel versão 2016. Os programas utilizados foram: EPIINFO versão 7 (Centers for Diseases Control and Prevention, Atlanta/Geórgia/Estados Unidos). Foram apresentados, descritivamente, por meio de representação tabular constando a frequência.

\subsection{Aprovação ética}

Esta pesquisa foi aprovada pela Comissão Científica COMIC - HU-UFMA, nº 48/2018.

\section{Resultados e Discussão}

Entre os setores do Hospital Universitário de São Luís do Maranhão, 61,2\% dos profissionais das equipes de enfermagem consideram a ala de internação pediátrica como a mais perigosa (Quadro 1), seguida pela área assistencial total $(57,2 \%)$, que inclui todos os setores que prestam assistência aos pacientes, e expurgo (20,4\%). Os principais riscos para os profissionais, nos três setores mencionados (internação, o expurgo e a área assistencial), estão relacionados aos perfurocortantes, contato com secreções e químicos, além de ruídos de máquinas e aparelhos.

Quadro 1 - Dados da Enfermagem Assistencial do Hospital Universitário de São Luís do Maranhão, segundo local que consideram mais perigoso em 2018. (N.98)

\begin{tabular}{|l|c|c|}
\hline $\begin{array}{c}\text { Quais os setores do Hospital Universitário } \\
\text { que você considera mais perigoso }\end{array}$ & N & \% \\
\hline A estrutura física & 9 & 9,2 \\
\hline A internação pediátrica (Ala A, Ala D, Ala E) & 60 & 61,2 \\
\hline Área assistencial total & 56 & 57,2 \\
\hline Expurgo & 20 & 20,4 \\
\hline Todos os setores do hospital & 6 & 6,1 \\
\hline
\end{tabular}

Fonte: Dados da pesquisa.

Outro fator de risco evidenciado pela literatura, porém não evidenciado nesta pesquisa é a violência psicológica, considerada um risco ocupacional, pois gera risco e agravo à saúde do trabalhador e compromete a prestação do serviço pelo profissional, que sofreu violência, afetando o processo do cuidar (SULZBACHER; FONTANA, 2013).

Quando questionados sobre as atividades realizadas pela equipe de enfermagem, que consideravam mais perigosas para a saúde e para o meio ambiente, os entrevistados destacaram o descarte de resíduo hospitalar $(33,7 \%)$, seguido pela manipulação dos resíduos dos pacientes e aspiração traqueal $(18,4 \%)$ e atividades nas quais os profissionais entram em contato com material perfurocortante $(14,3 \%)$, como a punção venosa (Quadro 2).
Quadro 2 - Dados da Enfermagem Assistencial do Hospital Universitário de São Luís do Maranhão, segundo atividade que a equipe de enfermagem realiza considerando mais perigosa para sua saúde e para o meio ambiente em 2018, (N.98)

\begin{tabular}{|l|c|c|}
\hline $\begin{array}{l}\text { Qual a atividade que a equipe de enfermagem } \\
\text { realiza que você percebe mais perigosa para } \\
\text { a sua saúde e para o ambiente hospitalar }\end{array}$ & N & \% \\
\hline A manipulação dos resíduos dos pacientes & 18 & 18,4 \\
\hline Administração e manipulação de fármacos & 7 & 7,1 \\
\hline Aspiração traqueal & 18 & 18,4 \\
\hline $\begin{array}{l}\text { Atividade em que entramos em contato com } \\
\text { material perfuro cortante como a punção } \\
\text { venosa e procedimentos com pacientes com } \\
\text { doenças infectocontagiosas }\end{array}$ & 29 & 14,3 \\
\hline Descarte de resíduo hospitalar & 33 & 33,7 \\
\hline $\begin{array}{l}\text { Toda e qualquer atividade desempenhada pelo } \\
\text { enfermeiro de UTI }\end{array}$ & 6 & 6,1 \\
\hline Não respondeu & 4 & 4,1 \\
\hline
\end{tabular}

Fonte: Dados da pesquisa.

Os profissionais percebem ser mais perigoso para a saúde o risco de contaminação através do descarte e manipulação dos resíduos, em que pode ocorrer acidentes com material perfurocortante, e disseminação de bactérias multirresistentes, que além de representar risco à saúde também causam prejuízos ao meio ambiente. Podendo haver mais de uma resposta por participante da pesquisa, para estas perguntas. Esta percepção do descarte de resíduos como risco ocupacional também foi observada por Justiniano et al. (2020), trabalho em que os entrevistados destacam a necessidade de manter práticas de manuseio adequado dos resíduos durante as atividades assistenciais.

De acordo com Januário et al. (2017), entre as exposições ocupacionais em situações de prestações de cuidado aos pacientes envolvendo acidentes por material biológico, potencialmente contaminado, estão a via percutânea $(60,7 \%)$, ferimentos ocorridos com instrumentos perfurocortantes, e a via mucocutânea (39,3\%), pele íntegra e não íntegra, exposição de mucosa ocular, nasal, oral, genital e mordeduras humanas. Diante dos estudos, os profissionais de enfermagem estão mais suscetíveis à ocorrência das exposições percutâneas, como fluído corporal, manuseio inadequado de materiais perfurocortantes, cateter intravenoso e materiais de punção venosa, em situações de prestação de cuidados ao paciente.

Conforme pode ser observado no Quadro 3, a estrutura física do hospital se destaca $(35,7 \%)$ entre as dificuldades encontradas pelas equipes de enfermagem para o descarte de resíduos, seguido por roupa que assegurasse uma maior proteção quando do descarte de secreção no expurgo $(30,6 \%)$ e pela escassez de caixas para descarte de perfurocortantes $(23,5 \%)$, que tem como consequência o seu uso acima do volume recomendado pelos fabricantes. Podendo haver mais de uma resposta por participante da pesquisa, para estas perguntas. 
Quadro 3 - Dados da Enfermagem Assistencial do Hospital Universitário de São Luís do Maranhão, segundo dificuldades que são observadas para o descarte dos resíduos no serviço de enfermagem em 2018.

\begin{tabular}{|l|c|c|}
\hline $\begin{array}{l}\text { Quais as dificuldades que você observa para o } \\
\text { descarte dos resíduos }\end{array}$ & $\mathbf{N}$ & $\mathbf{\%}$ \\
\hline A estrutura física do Hospital. & 35 & 35,7 \\
\hline $\begin{array}{l}\text { Roupa que assegurasse uma maior proteção } \\
\text { quando do descarte de secreção no expurgo. }\end{array}$ & 30 & 30,6 \\
\hline $\begin{array}{l}\text { Falta de caixa para perfurocortantes, a caixa fica } \\
\text { muito cheia. }\end{array}$ & 23 & 23,5 \\
\hline $\begin{array}{l}\text { A não disponibilidade de locais adequados } \\
\text { para cada tipo de resíduo em alguns pontos do } \\
\text { ambiente. }\end{array}$ & 22 & 22,4 \\
\hline $\begin{array}{l}\text { Acesso no expurgo para o descarte por conta da } \\
\text { demora do(a) colega. }\end{array}$ & 5 & 5,1 \\
\hline $\begin{array}{l}\text { Caixa para perfurocortantes em uma posição } \\
\text { muito elevada. }\end{array}$ & 2 & 2,0 \\
\hline Às vezes, lixeiras sem sacos. & 1 & 1,0 \\
\hline Não encontro dificuldades. & 1 & 1,0 \\
\hline Fonte: Dados da pesquisa. & & \\
\hline
\end{tabular}

A estrutura física do hospital teve a maior frequência de citações $(35,7 \%)$, por ser um espaço muito pequeno para os profissionais, o que dificulta o descarte de resíduos, aumentando o risco de ocorrência de acidentes com perfurocortantes. Outros destaques foram dados à roupa que assegurasse maior proteção $(30,6 \%)$, e falta de caixa para perfurocortantes $(23,5 \%)$. Nesse sentido, a enfermagem, por ser a categoria de maior quantidade e permanência hospitalar, se encontra, particularmente, exposta ao maior risco biológico, sendo o contato com material perfurocortante e sangue frequentes entre esses (MARTINS et al., 2014), além dos riscos físicos, químicos, mecânicos e psicossociais (PUCCI et al., 2017; MARTINS et al., 2020). Quando o trabalho é realizado em condições insalubres e inseguras, como o contato com riscos químicos, físicos, biológicos, ergonômicos e de acidentes, podem afetar o bem-estar físico e psíquico do indivíduo (JANUÁRIO et al., 2017). O que, por sua vez, evidencia a importância do estímulo à adoção da cultura de segurança no ambiente hospitalar (SILVA-BATALHA; MELLEIRO, 2015; BRASIL, 2017; SOARES et al., 2019).

Ao serem questionados sobre sugestões ou propostas para melhorar a segurança no ambiente de trabalho das equipes de enfermagem, podendo haver mais de uma resposta por participante da pesquisa para estas perguntas, conforme pode ser observado no Quadro 2, a maioria dos entrevistados sugere a realização de mais treinamentos com os profissionais $(27,6 \%)$, além de melhoria da estrutura física do Hospital (19,4\%), inclusive, com aumento do expurgo, tornando o ambiente mais amplo com maior mobilidade. O uso de EPIs em todos os procedimentos e, principalmente, respeitando as normas de segurança contra infecções visando segurança do paciente e, disponibilidade de lixeiras comuns e de contaminados, na mesma proporção nas enfermarias e corredores também foram sugestões de parcela expressiva entre os entrevistados, correspondendo à $18,4 \%$ e $13,4 \%$ respectivamente.

No tocante aos treinamentos com os profissionais, sugerido pela maioria dos entrevistados, Brand e Fontana (2014) sugerem a educação permanente em saúde aos trabalhadores para aperfeiçoar mecanismos que garantam a segurança e saúde destes sujeitos. As instituições promotoras de saúde devem se responsabilizar pela educação continuada de seus trabalhadores por meio da prevenção de acidentes de trabalho com a sensibilização e conscientização desses. Tais práticas devem estar presentes em todas as propostas de capacitação de pessoal, visando a segurança ocupacional (BRASIL, 2005; BRASIL, 2018). No tocante ao uso de EPIs, frequentemente, os trabalhadores de saúde manipulam instrumentos inseguros e não utilizam Equipamento de Proteção Individual adequado (SARQUIS; FELLI, 2009).

Questões como riscos ergonômicos e elevada carga horária de trabalho não foram mencionadas pelos entrevistados neste estudo, embora na literatura haja menção às repercussões negativas relacionadas aos riscos ergonômicos, que têm origem em fatores como a postura inadequada na realização de procedimentos, levantamentos de peso, tanto de pacientes quanto de equipamentos, os corredores longos, manipulação de cargas, mobílias insuficientes e inadequadas para a postura laboral, entre outros(SHOJIetal.,2015; MARTINS etal.,2020).

Quadro 4 - Segundo sugestões ou propostas para melhorar a segurança para o serviço de enfermagem em 2018

\begin{tabular}{|l|c|c|}
\hline $\begin{array}{l}\text { Você tem sugestões ou propostas para } \\
\text { melhorar a segurança para o serviço de } \\
\text { enfermagem }\end{array}$ & N & \% \\
\hline Mais treinamentos com os profissionais. & 27 & 27,6 \\
\hline $\begin{array}{l}\text { Melhorar a estrutura física do Hospital: } \\
\text { aumentando o expurgo, tornando o ambiente } \\
\text { mais amplo que nos proporcione mais } \\
\text { mobilidade, }\end{array}$ & 19 & 19,4 \\
\hline $\begin{array}{l}\text { Uso de EPI's em todos os procedimentos e, } \\
\text { principalmente, respeitando as normas de } \\
\text { segurança contra infecçoses visando segurança } \\
\text { do paciente. }\end{array}$ & 18 & 18,4 \\
\hline $\begin{array}{l}\text { A mesma quantidade de lixeiras comuns nas } \\
\text { enfermarias e corredores. }\end{array}$ & 13 & 13,2 \\
\hline $\begin{array}{l}\text { Mais materiais e equipamentos para o trabalho } \\
\text { e orientações para os acompanhantes. }\end{array}$ & 8 & 8,2 \\
\hline $\begin{array}{l}\text { Atividades educativas (palestras, dinâmica de } \\
\text { grupo), conscientização da equipe e materiais } \\
\text { em quantidade suficiente para a realização da } \\
\text { assistência. }\end{array}$ & 7 & 7,2 \\
\hline $\begin{array}{l}\text { Coleta rigorosa dos resíduos contaminados nos } \\
\text { postos e enfermarias. }\end{array}$ & 5 & 5,2 \\
\hline $\begin{array}{l}\text { Melhora na qualidade dos equipamentos e } \\
\text { produtos oferecidos. }\end{array}$ & 4 & 4,1 \\
\hline $\begin{array}{l}\text { Aumentar a quantidade de profissionais na } \\
\text { equipe, em função de sobrecarga de trabalho. }\end{array}$ & 4 & 4,1 \\
\hline A liderança ouvir melhor a equipe de trabalho. & 1 & 1,0 \\
\hline
\end{tabular}

Fonte: Dados da pesquisa.

No tocante à elevada carga horária de trabalho, esta tem como consequência um processo de desgaste revelado pela 
perda total ou parcial da capacidade corporal e psíquica, englobando os processos biopsíquicos (ARAÚJO, 2015). Importante destacar que reconhecer os riscos ocupacionais, que os profissionais da enfermagem estão sujeitos e em massa sofrem, é o primeiro passo para elaborar medidas que venham prevenir, amenizar e, se possível, sanar os riscos, como bemdito por Martins et al. (2020).

\section{Conclusão}

Os riscos biológicos são percebidos com mais evidência pelos profissionais de enfermagem, embora reconheçam a existência de outros riscos.

As formas de prevenção e tomadas de atitudes necessárias para a melhoria da segurança no ambiente de trabalho indicadas em maior frequência pelos participantes da pesquisa foram: aumento da oferta de treinamentos em serviço; melhoria da estrutura física do Hospital, com aumento do expurgo; uso de EPIs adequados por todos os funcionários na execução de procedimentos.

Os riscos ambientais também são evidenciados pela indicação da necessidade de aumento da quantidade de caixas para descarte de material perfurocortante. Nesse contexto, espera-se, com este estudo, contribuir para minimizar os riscos a que os profissionais de enfermagem estão expostos, por meio da sensibilização das equipes e dos gestores do Hospital.

\section{Referências}

ARAÚJO, S.N.P. Os riscos enfrentados pelos profissionais de enfermagem no exercício da atividade laboral. Rev. Enferm. Contemp., v.4, n.2, p.237-243, 2015. doi: 10.17267/23173378rec.v4i2.522

BAKKE, H.A.; ARAÚJO, N.M.C. Acidentes de trabalho com profissionais de saúde de um hospital universitário. Rev Prod., v.20, n.4, p.669-676, 2010. doi: 10.1590/S0103-65132010005000015.

BRAND, C. I.; FONTANA, R. T. Biossegurança na perspectiva da equipe de enfermagem de unidades de tratamento intensivo. Rev. Bras. Enferm., v.67, n.1, p.78-84, 2014. doi: 10.5935/00347167.20140010.

BRASIL. Ministério do Trabalho e Emprego. Norma Regulamentadora $n^{\circ}$. 32. Segurança e Saúde no Trabalho em Estabelecimentos de Saúde. Brasília: Ministério do Trabalho e Emprego, 2005.

BRASIL. Resolução $n^{\circ}$ 466, de 12 de dezembro de 2012. Dispõe sobre diretrizes e normas

regulamentadoras de pesquisas envolvendo seres humanos. Diário Oficial [da] República Federativa do Brasil, 2013. Disponível em: $<$ https://conselho.saude.gov.br/resolucoes/2012/Reso466.pdf > Acesso em: 7 abr. 2020.

BRASIL. Ministério da Saúde. Portaria de consolidação $n^{\circ} 5$, de 28 de setembro de 2017. Consolidação das normas sobre as ações e os serviços de saúde do Sistema Único de Saúde. 2017.

BRASIL. Ministério da Saúde. Agência Nacional de Vigilância Sanitária. Gerência de Regulamentação e Controle Sanitário em Serviços de Saúde. Gerência Geral de Tecnologia em Serviços de Saúde. RDC n 222/2018 COMENTADA. 2018.

FURTADO, B.M.A.S.M; ARAÚJO JÚNIOR, J.L.C.Percepção de enfermeiros sobre condições de trabalho em setor de emergência de um hospital. Acta Paul. Enferm., v.23, n.2, p.169-174, 2010. doi: 10.1590/S0103-21002010000200003

GASPARINO, R.C. et al. Percepção da enfermagem frente ao clima de segurança do paciente em instituições públicas e privadas. Rev. Gaúcha, v.38, n.3, p.1-8, 2017. doi: 10.1590/19831447.2017.03.68240

JANUÁRIO, G. C. et al. Acidentes ocupacionais com material potencialmente contaminado envolvendo trabalhadores de enfermagem. Cogitare Enferm., v.22, n.1, p.1-9, 2017. doi: 10.5380/ce.v22i1.48893

JUSTINIANO, G.P.M. et al. Riscos ocupacionais e os resíduos de serviços de saúde em centro cirúrgico. Rev. SOBECC, v.25, n.1, p.25-32, 2020. doi: 10.5327/Z1414-4425202000010005

LEITE, J.W.; ARAUJO, G.F. Riscos ocupacionais: percepção de enfermeiros de um hospital público. Rev. Enferm. Contemp., v.5. n.2, p.201-209, 2016. doi: 10.17267/2317-3378rec.v5i2.1055

MARTINS, J. T. et al. Equipe de enfermagem de emergência: riscos ocupacionais e medidas de autoproteção. Rev. Enferm. UERJ, v. 22, n. 3, p. 334-340, 2014.

MARTINS, K. F.; ALVES, M.S.; DIAS A.K. Qualidade de vida no ambiente hospitalar dos profissionais de enfermagem. Rev Amaz. Health, v.8, n.1, p.37-54, 2020. doi: 10.18606/2318-1419/ amazonia.sci.health.v8n1p37-54

NISHIDE, V.M; BENATTI, M.C.C; ALEXANDRE, N.C. Ocorrência de acidente do trabalho em uma unidade de terapia intensiva. Rev. Latinoam. Enferm., v.12, n.2, p.204-211, 2004. doi: 10.1590/S0104-11692004000200009

PUCCI, S.M.; KANAN, L.A.; SILVA, B.F. Riscos psicossociais no contexto do trabalho. Rev. Gepes Vida, v. 3, n. 6, p.142-153, 2017.

ROCHA, F.L.R; MARZIALE, M.H.P.; ROBAZZI, M.L.C.C. Perigos potenciais a que estão expostos os trabalhadores de enfermagem na manipulação de quimioterápicos antineoplásicos: conhecê-los para preveni-los. Rev. Latinoam. Enferm., v.12, n.3, p.511-517, 2004. doi: 10.1590/S0104-11692004000300009

SARQUIS, L.M.M; FELLI, V.E.A. Os sentimentos vivenciados após exposição ocupacional entre trabalhadores de saúde: fulcro para repensar o trabalho em instituições de saúde. Rev. Bras. Enferm., v.62, n.5, p.701-704, 2009. doi: 10.1590/S003471672009000500008

SHOJI, S.; SOUZA, N.; FARIAS, S.P. Impacto do ambiente laboral no processo saúde doença dos trabalhadores de enfermagem de uma unidade ambulatorial especializada. Rev. Mineira Enferm., v.19, n.1, p.43-48, 2015. 10.5935/1415-2762.20150004

SILVA-BATALHA, E.M.S.; MELLEIRO, M.M. Cultura de segurança do paciente em um hospital de ensino: diferenças de percepção existentes nos diferentes cenários dessa instituição. Texto Contexto Enferm., v.24, n.2, p.432-41, 2015. doi: 10.1590/0104-07072015000192014

SOARES, et al. Análise dos acidentes de trabalho com exposição a material biológico notificados por profissionais da saúde. Rev. Bras. Med. Trab., v.17, n.2, p.201-208, 2019.doi: 10.5327/ Z1679443520190341

SULZBACHER, E.; FONTANA, R. Concepções da equipe de enfermagem sobre a exposição a riscos físicos e químicos no ambiente hospitalar. Rev. Bras. Enferm., v.66, n.1, p.25-30, 2013. doi: 10.1590/S0034-71672013000100004

TALHAFERRO, B.; BARBOZA, D.B.; OLIVEIRA, A.R. Adesão ao uso dos equipamentos de proteção individual pela enfermagem. Rev. Ciênc. Méd., v.17, n.3-6, p.157-166, 2008. 